R. Szőke

Nagoya Math. J.

Vol. 154 (1999), 171-183

\title{
ADAPTED COMPLEX STRUCTURES AND GEOMETRIC QUANTIZATION
}

\author{
RÓBERT SZŐKE
}

\begin{abstract}
A compact Riemannian symmetric space admits a canonical complexification. This so called adapted complex manifold structure $J_{A}$ is defined on the tangent bundle. For compact rank-one symmetric spaces another complex structure $J_{S}$ is defined on the punctured tangent bundle. This latter is used to quantize the geodesic flow for such manifolds. We show that the limit of the push forward of $J_{A}$ under an appropriate family of diffeomorphisms exists and agrees with $J_{S}$.
\end{abstract}

\section{$\S 0$. Introduction}

Different kind of geometric constructions naturally lead to complex structures defined on tangent bundles. The main purpose of this paper is to explore the relationship of two such constructions.

The first of these is the so called adapted complex structure and the second comes from geometric quantization.

Adapted complex structures (see Section 2 for the precise definition) were discovered by studying certain global solutions of the complex, homogeneous Monge-Ampère equation on Stein manifolds. One starts with a compact, real-analytic Riemannian manifold $(M, g)$. The adapted complex structure lives on $T M$ or perhaps only on a subdomain $T^{r} M$ consisting of those vectors having length smaller than $r$ for some fixed positive radius $r$.

These complex manifolds have many interesting properties. Among others the norm-square function is strictly plurisubharmonic and thus it defines a Kähler metric. The Kähler form of this metric is the symplectic form $\Omega$ of the tangent bundle (that comes from the canonical symplectic structure of the cotangent bundle using the metric to identify these two bundles).

The second kind of complex structures $J_{S}$ live on the punctured tangent bundle of compact, rank-1 symmetric spaces. For the spheres it was Souriau

Received May 6, 1998.

Research partially supported by the Hungarian Scientific Foundation (OTKA) under grant T21151. 
who in [So] identified the regularized Kepler manifold with the cotangent bundle of the sphere minus its zero section $\left(\stackrel{\circ}{T}^{*} S^{n}\right)$. He also defined a complex manifold structure on this space identifying it with the singular affine hyperquadric.

Later it was observed by Rawnsley [Ra1], that the norm function is strictly plurisubharmonic with respect to the above complex structure and thus defines a Kähler metric on $\stackrel{\circ}{T} S^{n}$ and the Kähler form is again $\Omega$. He also observed the complex structure to be invariant w.r.t. the normalized geodesic flow that he used in [Ra2] to quantize the geodesic flow on the spheres.

Subsequently Furutani and Tanaka [F-T] defined a Kähler structure on the punctured cotangent bundle of complex and quaternionic projective spaces. Their Kähler structure is also invariant w.r.t. the normalized geodesic flow. Furutani and Yoshizawa $[\mathrm{F}-\mathrm{Y}]$ used this Kähler structure to quantize the geodesic flow on complex and quaternionic projective spaces.

Furutani and Tanaka described their Kähler structures in terms of matrices in the spirit of Lie groups. A more geometric description of the complex (Kähler) structure on $\stackrel{\mathrm{T}}{ } M$ (where $M$ now can be either a sphere, a complex or quaternionic projective space or their quotient w.r.t. a discrete group of isometries) was given by Kiyotaka Ii [Ii]. In this paper we shall use Ii's description of these structures (see Section 3 for more details).

The main result of this paper (Theorem 3.2) is to show that for compact, rank-1 symmetric spaces the family of complex structures obtained by pushing forward the adapted complex structure with respect to an appropriate family of diffeomorphisms has a limit and this limit complex structure coincides with $J_{S}$.

After this paper was finished, we received a preprint from R. Aguilar $[\mathrm{Ag}]$, discussing, among other things, similar limits. Instead of the punctured tangent bundle, he works on the unit sphere bundle.

\section{$\S 1$. Geometry of the tangent bundle}

Let $M$ be a smooth $n$-dimensional manifold and $T M$ its tangent bundle. $\pi$ will denote the projection map $\pi: T M \rightarrow M$. We shall denote by $\stackrel{\circ}{\mathrm{T}} M$ the punctured tangent bundle i.e., $\stackrel{\circ}{\mathrm{T}} M=T M \backslash$ zero section $\}$.

Suppose a Riemannian metric $g$ is also given on $M$. The metric identifies the tangent and cotangent bundles and thus we can also talk about the 
canonical 1-form $\theta$ on $T M$, that is defined by

$$
\theta(v):=g\left(z, \pi_{*} v\right), \quad v \in T_{z}(T M)
$$

$\Omega:=d \theta$ defines then the symplectic form of $T M$.

When $M=\mathbb{R}$ we can canonically identify $T \mathbb{R}$ with the complex plane:

$$
T_{\sigma} \mathbb{R} \ni \tau \frac{\partial}{\partial \sigma} \stackrel{\iota}{\longmapsto} \sigma+i \tau \in \mathbb{C}
$$

From now on we shall always tacitly assume this identification. Denote by $\mathcal{H}_{+}$the upper half plane and by $T^{+} \mathbb{R}$ the corresponding part of $T \mathbb{R}$. For simplicity, we shall also assume that our manifold $(M, g)$ is complete.

Let $\gamma: \mathbb{R} \rightarrow M$ be a geodesic and $\gamma_{*}: T \mathbb{R} \rightarrow T M$ the induced map. As $\gamma$ runs through all geodesics, the images $\gamma_{*}\left(T^{+} \mathbb{R}\right)$ define a foliation of $\stackrel{\circ}{\mathrm{T}} M$. This foliation is called the Riemann foliation. We shall denote the corresponding distribution on $\stackrel{\circ}{\mathrm{T}} M^{\mathrm{by}} \mathcal{L}$.

Let $\rho: T M \rightarrow \mathbb{R}$ be the norm-square function. There is another natural distribution on $\stackrel{\circ}{\mathrm{T}} M$, denoted by $\mathcal{H}$, that at a point $z \in \stackrel{\circ}{\mathrm{T}} M$ is defined by

$$
\mathcal{H}_{z}:=\operatorname{ker} \theta_{z} \cap \operatorname{ker} d \rho
$$

$\mathcal{H}$ and $\mathcal{L}$ are complementary distributions, i.e., $T_{z}(\stackrel{\circ}{\mathrm{T}} M)=\mathcal{L}_{z}+\mathcal{H}_{z}$.

For a real number $s$ define the map $N_{s}: T M \rightarrow T M$ by multiplying every vector in each fibre with $s$. For a non-zero $s, N_{s}$ is a diffeomorphism.

The metric $g$ determines the Levi-Cività connection on $T M$ and thus a splitting of $T_{z}(T M)$ into horizontal and vertical subspaces. Let $v, z \in T_{p} M$. We shall denote the horizontal (resp. vertical) lift of the vector $v$ to $z$ by $\xi_{z}^{v} \in T_{z}(T M)\left(\operatorname{resp} . \eta_{z}^{v} \in T_{z}(T M)\right)$.

For an $\lambda \neq 0$ we get

$$
\begin{aligned}
& \left(N_{\lambda}\right)_{*} \xi_{z}^{v}=\xi_{\lambda z}^{v} \\
& \left(N_{\lambda}\right)_{*} \eta_{z}^{v}=\lambda \eta_{\lambda z}^{v}
\end{aligned}
$$

Now let $\gamma$ be a unit speed geodesic. Let $z=\tau_{0} \dot{\gamma}\left(\sigma_{0}\right), v=\dot{\gamma}\left(\sigma_{0}\right)$. Then we have

$$
\begin{aligned}
& \left(\gamma_{*}\right)_{*}\left(\left.\frac{\partial}{\partial \sigma}\right|_{\left(\sigma_{0}, \tau_{0}\right)}\right)=\xi_{z}^{v}, \\
& \left(\gamma_{*}\right)_{*}\left(\left.\frac{\partial}{\partial \tau}\right|_{\left(\sigma_{0}, \tau_{0}\right)}\right)=\eta_{z}^{v} .
\end{aligned}
$$




\section{$\S 2 . \quad$ Adapted complex structures}

For an $0<r \leq \infty$ denote by $T^{r} M$ the tube of vectors having length smaller than $r$.

Definition 2.1. Let $(M, g)$ be a complete Riemannian manifold. A smooth complex structure on $T^{r} M$ is called adapted if for any geodesic $\gamma: \mathbb{R} \rightarrow M$, the map

$$
\gamma_{*}:\left(\gamma_{*}\right)^{-1}\left(T^{r} M\right) \longrightarrow T^{r} M
$$

is holomorphic, where $\left(\gamma_{*}\right)^{-1}\left(T^{r} M\right) \subset T \mathbb{R}$ and $T \mathbb{R}$ is endowed with the complex structure from (1.2).

The adapted complex structure (if it exists) is unique and when $(M, g)$ is compact, real-analytic, we have existence for small enough $r$ (see [G-S], $[\mathrm{L}-\mathrm{Sz}],[\mathrm{Sz}])$.

One particular example that shall concern us in Section 3 is the case of the round sphere. The standard realization of $T S^{n}$ is

$$
\left\{(e, X) \in \mathbb{R}^{n+1} \times \mathbb{R}^{n+1} \mid\|e\|=1,\langle e, X\rangle=0\right\} .
$$

Let $X_{1}, X_{2} \in S^{n},\left\langle X_{1}, X_{2}\right\rangle=0$. Then $\gamma: \mathbb{R} \rightarrow S^{n} \subset \mathbb{R}^{n+1}, \gamma(\theta)=\cos \theta X_{1}+$ $\sin \theta X_{2}$ is a unit speed geodesic (i.e., a great circle) of $S^{n}$. Complexifying this map gives $\gamma_{\mathbb{C}}: \mathbb{C} \rightarrow \mathbb{C}^{n+1}$,

$$
\gamma_{\mathbb{C}}(\zeta)=\cos \zeta X_{1}+\sin \zeta X_{2}
$$

The image $\gamma_{\mathbb{C}}(\mathbb{C})$ lies in the affine hyperquadric $Q_{1} \subset \mathbb{C}^{n+1}$.

$$
\begin{aligned}
Q_{1} & =\left\{\left(z_{1}, \ldots, z_{n+1}\right) \in \mathbb{C}^{n+1} \mid \sum_{j=1}^{n+1} z_{j}^{2}=1\right\} \\
& =\left\{X+i Y \mid X, Y \in \mathbb{R}^{n+1},\langle X, X\rangle-\langle Y, Y\rangle=1,\langle X, Y\rangle=0\right\} .
\end{aligned}
$$

If we can find a diffeomorphism $\delta: T S^{n} \rightarrow Q_{1}$ such that the following diagram commutes, the pullback complex structure on $T S^{n}$ will be adapted $(\iota$ is from $(1.2))$.

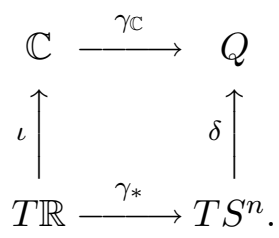


A little bit of calculation gives that the choice

$$
\delta(e, X):=\cosh (\|X\|) e+i \frac{\sinh (\|X\|)}{\|X\|} X
$$

will work.

It is always possible to express the almost complex tensor $J_{A}$ of the adapted complex structure in terms of Jacobi fields and analytic continuation (see [L-Sz]). On a symmetric space Jacobi's equation can be explicitly solved. This suggests that for such spaces the formula for $J_{A}$ should be very explicit as well. This is indeed the case.

Denote by $\mathcal{R}$ the curvature tensor of the metric and let $z \in T_{p} M$. The operator $R_{z}(\cdot)=\mathcal{R}(\cdot, z) z$ is the Jacobi operator associated to $z$. In fact a little bit of calculation together with [Sz, Theorem 2.5] yields:

Proposition 2.2. Let $(M, g)$ be a compact symmetric space (or a locally symmetric quotient of such). Let $z \in \stackrel{\circ}{\mathrm{T}} M, v_{1}=z /\|z\|$. Let $v_{1}, v_{2}, \ldots, v_{n}$ be an orthonormal basis of $T_{\pi z} M$ consisting of eigenvectors of the Jacobi operator $R_{v_{1}}$ with eigenvalue $\Lambda_{j}, j=1, \ldots, n$. Let $h(x):=x \operatorname{coth} x$. Let $\xi_{z}^{v_{j}}$ (resp. $\eta_{z}^{v_{j}}$ ) be the horizontal (resp. vertical) lift of $v_{j}$ to the point $z$, $j=1, \ldots, n$. Then

$$
J_{A} \xi_{z}^{v_{j}}=h\left(\sqrt{\Lambda_{j}}\|z\|\right) \eta_{z}^{v_{j}}
$$

i.e., with the positive, real-analytic function $t(x):=h(\sqrt{x})$, the matrix of $J_{A}$ in the basis $\xi_{z}^{v_{j}}, \eta_{z}^{v_{j}}, j=1, \ldots, n$ is:

$$
\left.J_{A}\right|_{z}=\left[\begin{array}{cc}
0 & -\left(t\left(R_{z}\right)\right)^{-1} \\
t\left(R_{z}\right) & 0
\end{array}\right]
$$

\section{§3. Complex structures on $\stackrel{\circ}{\mathrm{T}} M$}

In this section we discuss the complex structures on the punctured tangent bundles of compact, rank-1 symmetric spaces that arose in geometric quantization, using Ii's description of these structures. We start first with the spheres. We identify (using the metric) the tangent and cotangent bundles and take the standard representation (2.1) of the punctured tangent bundle of $S^{n}$. The singular affine hyperquadric $Q_{0}$ is defined as

$$
Q_{0}=\left\{z=\left(z_{1}, \ldots, z_{n+1}\right) \in \mathbb{C}^{n+1} \mid \sum_{j=1}^{n+1} z_{j}^{2}=0\right\}
$$


The complex structure $J_{S}$ on $\stackrel{\circ}{\mathrm{T}} S^{n}$ is obtained by identifying it with $Q_{0}$. More precisely, the map $\stackrel{\circ}{\mathrm{T}} S^{n} \rightarrow Q_{0} \backslash\{0\}$ defined by

$$
(e, X) \longmapsto z=\|X\| e+i X
$$

is a diffeomorphism. The pull back gives us the desired complex structure on $\stackrel{\circ}{\mathrm{T}} S^{n}$.

Ii's description of $J_{S}$ on $\stackrel{\circ}{\mathrm{T}} S^{n}$ is as follows. For $0 \neq z \in T_{p} S^{n}, v \in T_{p} M$, denote by $\xi_{z}^{v}$ (resp. $\eta_{z}^{v}$ ) the horizontal (resp. vertical) lift of the vector $v$ to the point $z$. Then

$$
J_{S} \xi_{z}^{v}=\|z\| \eta_{z}^{v}
$$

Our first observation about $J_{A}$ (the adapted complex tensor) and $J_{S}$ is that they are genuinely different complex structures on $\stackrel{\circ}{\mathrm{T}} S^{n}$.

Proposition 3.1. The complex manifolds $\left(\stackrel{\circ}{\mathrm{T}} S^{n}, J_{A}\right)$ and $\left(\stackrel{\mathrm{T}}{\mathrm{T}} S^{n}, J_{S}\right)$ are not biholomorphic.

Proof. The statement is equivalent to showing that $X=Q_{0} \backslash\{0\}$ and $Y=Q_{1} \backslash S^{n}$ (where $Q_{1}$ is the affine hyperquadric (see (2.2)) are not biholomorphic. Suppose on the contrary that $\psi: Y \rightarrow X$ is a biholomorphism. We can apply a theorem of Hartogs (see [Sh]), to conclude that $\psi$ has a holomorphic extension $\tilde{\psi}: Q_{1} \rightarrow \mathbb{C}^{n+1}$ (the theorem we use here says that if $N$ is a complex manifold, $L \subset N$ a real submanifold of real codimension at least 2 and $\psi: N \backslash L \rightarrow \mathbb{C}$ is holomorphic which does not extend holomorphically to a point $q \in L$, then in a neighborhood of $q, L$ is a complex submanifold).

Since $Q$ is defined as the zero set of a holomorphic function, therefore $\tilde{\psi}$ also maps into $Q_{0}$. Since $\psi$ is biholomorphic and $\tilde{\psi}$ is holomorphic, $\tilde{\psi}\left(S^{n}\right)$ must be the point 0 . But $S^{n}$ is a maximal dimensional totally real submanifold in $Q$, hence $\tilde{\psi}$ must be constant, a contradiction.

Despite of the negative result of Proposition 3.1, $J_{A}$ and $J_{S}$ do have something to do with each other. To discover their relation, consider now for any $t \geq 0$ the quadric

$$
Q_{t}=\left\{z \in \mathbb{C}^{n+1} \mid \sum_{j=1}^{n+1} z_{j}^{2}=t\right\}
$$

For $t, s>0, Q_{t}$ and $Q_{s}$ are biholomorphic complex submanifolds and $Q_{t} \backslash \mathbb{R}^{n}$ and $Q_{s} \backslash \mathbb{R}^{n}$ are both biholomorphic to $\left(\stackrel{\mathrm{T}}{\mathrm{T}} S^{n}, J_{A}\right)$. The complex manifold 
$Q_{0} \backslash \mathbb{R}^{n}=Q_{0} \backslash\{0\}$ is in some sense the limit of the complex manifolds $Q_{t} \backslash \mathbb{R}^{n}$ when $t$ goes to zero. This gives the idea to try to push forward $J_{A}$ by a family of diffeomorphisms in such a way that the limit of these push forwards is $J_{S}$. This indeed can be done as the following theorem shows.

TheOREM 3.2. Let $(M, g)$ be a compact, rank-1 symmetric space. Denote by $\Phi_{\varepsilon}$ the diffeomorphism $: \stackrel{\circ}{\mathrm{T}} M \rightarrow \stackrel{\mathrm{T}}{\mathrm{T}} M$

$$
\Phi_{\varepsilon}(v)=\varepsilon \exp (\|v\|) \frac{v}{\|v\|} .
$$

Then the following limit complex structure $J_{0}$ exits

$$
\lim _{\varepsilon \rightarrow 0}\left(\Phi_{\varepsilon}\right)_{*} J_{A}=J_{0}
$$

The maps $N_{s}, s \neq 0$ and the normalized geodesic flow are $J_{0}$ holomorphic. When $M$ is the sphere, $J_{0}$ agrees with $J_{S}$ and for the complex and quaternionic projective spaces it coincides with the structure studied by FurutaniTanaka-Ii ([F-T], [Ii] $)$.

Remark. Since the entire construction and the proof is compatible with taking quotients w.r.t. a discrete group of the isometry group, the result of Theorem 3.2 is valid for such quotients as well.

When $(M, g)$ is a round sphere, it was already noticed by Su-Jen Kan in $[\mathrm{Ka}]$, that the CR-manifolds $S_{r}=\{\|v\|=r\}$ posses a limit CR structure as $r \rightarrow \infty$.

Before we go on with the proof, we give another description of the complex structures studied in [F-T], [Ii], [Ra1], [So]. Recall the notion of Jacobi operator $R_{z}$ from Section 2. $R_{z}$ is self-adjoint and $z$ always belongs to its kernel. We shall need a modified operator $\widetilde{R}_{z}: T_{p} M \rightarrow T_{p} M$, defined as follows

$$
\widetilde{R}_{z}(X)=\langle X, z\rangle z+R_{z}(X)=\langle X, z\rangle z+\mathcal{R}(X, z) z .
$$

Define the almost complex tensor $J_{0}: T_{z}(T M) \rightarrow T_{z}(T M)$ in the horizontal and verical decomposition $T_{z}\left(T S^{n}\right)=H_{z}+V_{z}$, by the formula

$$
J_{0}=\left[\begin{array}{cc}
0 & -\left(\sqrt{\widetilde{R}_{z}}\right)^{-1} \\
\sqrt{\widetilde{R}_{z}} & 0
\end{array}\right] .
$$


EXAMPLE.

1) Let $(M, g)$ have constant curvature 1 . Then the curvature tensor $\mathcal{R}$ has a particularly simple form $\mathcal{R}(X, Y) Z=\langle Y, Z\rangle X-\langle X, Z\rangle Y$.

Therefore in this case $\widetilde{R}_{z}(X)=\langle z, z\rangle X$. This implies that for $z \neq 0$ the complex structure $J_{S}$ of $(3.2)$, agrees with $J_{0}$.

2) Let now $(M, g, J)$ be a Kähler manifold of constant holomorphic sectional curvature 4 . Then the curvature tensor has the following form

$$
\begin{gathered}
\mathcal{R}(X, Y) Z=\langle Y, Z\rangle X-\langle X, Z\rangle Y-\langle Y, J Z\rangle J X \\
+\langle X, J Z\rangle J Y+2\langle X, J Y\rangle J Z
\end{gathered}
$$

This yields

$$
\widetilde{\mathcal{R}}_{z} v=\langle z, z\rangle v+3\langle v, J z\rangle J z
$$

From this it follows:

$$
\sqrt{\widetilde{\mathcal{R}}_{z}} v=\frac{\langle v, J z\rangle}{\|z\|} J z+\|z\| v, \quad z \neq 0 .
$$

The complex structure on $\mathrm{T}^{\mathrm{T}} M$ studied by Furutani and Tanaka in $[\mathrm{F}-\mathrm{T}]$ is defined by (see [Ii])

$$
J_{1}\left(\xi_{z}^{v}\right)= \begin{cases}2\|z\| \eta_{z}^{v}, & \text { if } v=\alpha J z, \text { for some } \alpha \in \mathbb{R} \\ \|z\| \eta_{z}^{v}, & \text { if } v \perp J z\end{cases}
$$

It follows from formula (3.6) that $J_{1}$ in fact has the form of (3.5).

$3)$ Let $\left(M, g, I_{1}, I_{2}, I_{3}\right)$ be a quaternion Kähler manifold of constant Q-sectional curvature 4. Then

$$
\begin{aligned}
\mathcal{R}(X, Y) Z=\langle Y, Z\rangle X-\langle X, Z\rangle Y & \\
& -\sum_{i=1}^{3}\left\langle Y, I_{i} Z\right\rangle I_{i} X+\left\langle X, I_{i} Z\right\rangle I_{i} Y+2\left\langle X, I_{i} Y\right\rangle I_{i} Z .
\end{aligned}
$$

This yields

$$
\widetilde{\mathcal{R}}_{z} v=\langle z, z\rangle v+\sum_{i=1}^{3}\left\langle v, I_{i} z\right\rangle I_{i} z
$$

Thus

$$
{\sqrt{\widetilde{\mathcal{R}}_{z}}}_{v} v=\|z\| v+\sum_{i=1}^{3} \frac{\left\langle v, I_{i} z\right\rangle}{\|z\|} I_{i} z
$$


The complex structure of Furutani-Tanaka is defined on $\mathrm{T}^{\mathrm{T}} M$ by (see [Ii])

$$
J_{2} \xi_{z}^{v}= \begin{cases}2\|z\| \eta_{z}^{v}, & \text { if } v=\sum_{i=1}^{3} \alpha_{i} I_{i} z \text { for some } \alpha_{i} \in \mathbb{R} \\ \|z\| \eta_{z}^{v}, & \text { if } v \perp I_{i} z, i=1,2,3\end{cases}
$$

Comparing this to formula (3.7) yields that $J_{2}$ again has the form (3.5).

Remark. The formula (3.5) for $J_{0}$ is so simple that it cries for a generalization for higher rank symmetric spaces. Of course when the rank is larger than 1 , the operators $\sqrt{\widetilde{R}_{z}}$ are no longer invertible, so formula (3.5) makes no sense literaly. But an appropriate formulation of Theorem 3.2 is still valid. We shall discuss these circle of questions in another publication.

Proof of Theorem 3.2. We shall prove that $\lim _{\varepsilon \rightarrow 0}\left(\Phi_{\varepsilon}\right)_{*} J_{A}$ exists and has the form of (3.5).

STEP 1.

The diffeomorphisms $\Phi_{\varepsilon}$ map any leaf of the Riemann foliation onto itself. First we shall prove the existence of the limit complex structure along a leaf.

Let $\gamma: \mathbb{R} \rightarrow M$ be a unit speed geodesic. Denote by $\mathcal{H}_{+}$the upper half plane. Then $\gamma_{*}: \mathcal{H}_{+} \rightarrow T M,(\sigma+i \tau) \mapsto \tau \dot{\gamma}(\sigma)$ parametrizes a leaf. Define $\psi_{\varepsilon}: \mathcal{H}_{+} \rightarrow \mathcal{H}_{+}$by $\psi_{\varepsilon}(\sigma+i \tau)=\sigma+i \varepsilon \exp \tau$. Then $\psi_{\varepsilon}$ is a diffeomorphism of $\mathcal{H}_{+}$and the following diagram commutes:

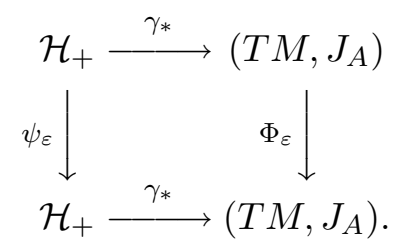

Denote by $J_{+}$the complex tensor on $\mathcal{H}_{+}$. Since $\gamma_{*}$ is holomorphic, we get

$$
\left(\Phi_{\varepsilon}\right)_{*} J_{A}=\left(\Phi_{\varepsilon}\right)_{*}\left(\gamma_{*}\right)_{*} J_{+}=\left(\gamma_{*}\right)_{*}\left(\psi_{\varepsilon}\right)_{*} J_{+}
$$

Now let $\sigma_{1}+i \tau_{1} \in \mathcal{H}_{+}$. It follows from the definition of $\psi_{\varepsilon}$, that

$$
\left(\psi_{\epsilon}\right)_{*}\left(\left.\partial \sigma\right|_{\sigma_{1}+i \tau_{1}}\right)=\left.\partial \sigma\right|_{\sigma_{1}+i \varepsilon \exp \tau_{1}}
$$

and

$$
\left(\psi_{\epsilon}\right)_{*}\left(\left.\partial \tau\right|_{\sigma_{1}+i \tau_{1}}\right)=\left.\varepsilon e^{\tau_{1}} \partial \tau\right|_{\sigma_{1}+i \varepsilon \exp \tau_{1}} .
$$


This implies that the push forward complex structure at a fixed point $\sigma_{0}+i \tau_{0}$ can be computed as follows

$$
\begin{aligned}
& {\left[\left(\psi_{\varepsilon}\right)_{*} J_{+}\right]\left(\left.\partial \sigma\right|_{\left(\sigma_{0}+i \tau_{0}\right)}\right)} \\
& \quad=\left(\psi_{\varepsilon}\right)_{*}\left(\left.J_{+}\right|_{\left(\sigma_{0}+i \log \left(\tau_{0} / \varepsilon\right)\right)}\left(\left.\partial \sigma\right|_{\left(\sigma_{0}+i \log \left(\tau_{0} / \varepsilon\right)\right)}\right)\right) \\
& \quad=\left(\psi_{\varepsilon}\right)_{*}\left(\left.\partial \tau\right|_{\left(\sigma_{0}+i \log \left(\tau_{0} / \varepsilon\right)\right.}\right) \\
& \quad=\left.\varepsilon \exp \left(\log \left(\tau_{0} / \varepsilon\right)\right) \partial \tau\right|_{\left(\sigma_{0}+i \tau_{0}\right)} \\
& \quad=\left.\tau_{0} \partial \tau\right|_{\left(\sigma_{0}+i \tau_{0}\right)}
\end{aligned}
$$

Let $Y=\dot{\gamma}\left(\sigma_{0}\right)$ and $X=\tau_{0} Y$. Recall from (1.5) that

$$
\left(\gamma_{*}\right)_{*}\left(\left.\partial \sigma\right|_{\left(\sigma_{0}+i \tau_{0}\right)}\right)=\xi_{X}^{Y}, \quad\left(\gamma_{*}\right)_{*}\left(\left.\partial \tau\right|_{\left(\sigma_{0}+i \tau_{0}\right)}\right)=\eta_{X}^{Y}
$$

Furthermore

$$
\widetilde{R}_{X}(Y)=\langle Y, X\rangle X
$$

hence

$$
\sqrt{\widetilde{R}_{X}} Y=\frac{\langle Y, X\rangle}{\|X\|} X=X
$$

So we get

$$
J_{0}\left(\left(\gamma_{*}\right)_{*}\left(\left.\partial \sigma\right|_{\left(\sigma_{0}+\tau_{0}\right)}\right)\right)=J_{0} \xi_{X}^{Y}=\eta_{X}^{X}=\left(\gamma_{*}\right)_{*}\left(\left.\tau_{0} \partial \tau\right|_{\left(\sigma_{0}+i \tau_{0}\right)}\right)
$$

(3.8), (3.10), (3.10) together imply that the image $\gamma_{*}\left(\mathcal{H}_{+}\right)$is a complex submanifold w.r.t. both structures, $J_{0}$ and $\left(\Phi_{\varepsilon}\right)_{*} J_{A}$ and in fact these two complex structures coincide.

\section{STEP 2.}

To complete the proof of the theorem we need to show the existence of the limit $(\Phi)_{*} J_{A}$, when we restrict ourselves to the distribution $\mathcal{H}$, the complementary distribution to $\mathcal{L}$ (see (1.3). Let $\varepsilon>0$ and $p \in \mathrm{T}^{\circ} M$ be fixed, $\|p\|=c$, define $v_{1}=p / c, r=\log (c / \varepsilon), q=(r / c) p, \lambda=\left(\varepsilon e^{r}\right) / r$. Then $\Phi_{\varepsilon}$ maps the hypersurface $S_{r}=\{u=r\}$ onto $S_{c}=\{u=c\}$.

As before denote by $N_{\lambda}$ the diffeomorphism of $T M$ that is multiplication by $\lambda$ in the fibers. It is clear that

$$
\left.\Phi_{\varepsilon}\right|_{S_{r}}=\left.N_{\lambda}\right|_{S_{r}}
$$


Choose eigenvectors $v_{2}, \ldots, v_{n}$ of $R_{v_{1}}$ such that together with $v_{1}$ they form an orthonormal basis for $T_{\pi(p)} M$. Recall from (2.4)

$$
J_{A} \xi_{q}^{v_{j}}=h\left(\sqrt{\Lambda_{j}} r\right) \eta_{q}^{v_{j}}
$$

where $h(x)=x \operatorname{coth} x$. From (1.4) we know

$$
\left(N_{\lambda}\right)_{*} \xi_{q}^{v_{j}}=\xi_{p}^{v_{j}}, \quad\left(N_{\lambda}\right)_{*} \eta_{q}^{v_{j}}=(c / r) \eta_{p}^{v_{j}} .
$$

From (3.11), (3.12) and (3.13) we get

$$
\begin{aligned}
\left(\Phi_{\varepsilon}\right) * J_{A} \xi_{p}^{v_{j}} & =\left(\Phi_{\varepsilon}\right)_{*}\left(J_{A}\left(\left(\Phi_{\varepsilon}\right)_{*}^{-1} \xi_{p}^{v_{j}}\right)\right) \\
& =\left(\Phi_{\varepsilon}\right)_{*}\left(J_{A} \xi_{q}^{v_{j}}\right) \\
& =\left(\Phi_{\varepsilon}\right)_{*}\left(h\left(\sqrt{\Lambda_{j}} r\right) \eta_{q}^{v_{j}}\right) \\
& =h\left(\sqrt{\Lambda_{j}} r\right)(c / r) \eta_{p}^{v_{j}}
\end{aligned}
$$

Now $r \rightarrow \infty$ as $\varepsilon \rightarrow 0$. Hence $h\left(\sqrt{\Lambda_{j}} r\right)(c / r)=\sqrt{\Lambda_{j}} \operatorname{coth}\left(\sqrt{\Lambda_{j}} r\right) c \rightarrow$ $\sqrt{\Lambda_{j}} c$. This together with (3.14) implies

$$
\lim _{\varepsilon \rightarrow 0}\left(\Phi_{\varepsilon}\right)_{*} J_{A} \xi_{p}^{v_{j}}=\sqrt{\Lambda_{j}} c \eta_{p}^{v_{j}}
$$

On the other hand

$$
\begin{aligned}
\widetilde{R}_{p} v_{j} & =\left\langle v_{j}, p\right\rangle p+R_{p}\left(v_{j}\right) \\
& =\left\langle v_{j}, c v_{1}\right\rangle c v_{1}+c^{2} R_{v_{1}} v_{j} \\
& =c^{2} \Lambda_{j} v_{j} .
\end{aligned}
$$

This implies

$$
J_{0} \xi_{p}^{v_{j}}=c \sqrt{\Lambda_{j}} \eta_{p}^{v_{j}} .
$$

Finally (3.15) and (3.16) together imply that indeed $\lim _{\varepsilon \rightarrow 0}\left(\Phi_{\varepsilon}\right)_{*} J_{A}$ exists and equals to $J_{0}$. The examples after (3.5) show that $J_{0}$ in fact coincides with the complex structure studied by Furutani-Tanaka-Ii. The normalized geodesic flow was shown to be $J_{0}$-holomorphic in [Ii].

Let now $0 \neq s, 0 \neq p \in T_{m} M$ and $v \in T_{m} M$ be arbitrary. Define $w$ by $w=\sqrt{\widetilde{R}_{s p}} v$. From the definition of $\widetilde{R}_{s p}$ we get that

$$
w=s \sqrt{\widetilde{R}_{p}} v
$$


This together with the action (1.4) of $\left(N_{s}\right)_{*}$ and the definition (3.5) of $J_{0}$ yields:

$$
\left.J_{0}\right|_{s p}\left(N_{s}\right)_{*} \xi_{p}^{v}=\left.J_{0}\right|_{s p} \xi_{s p}^{v}=\xi_{s p}^{w}=\left(N_{s}\right)_{*} J_{p} \xi_{p}^{v}
$$

i.e., $N_{s}$ is indeed $J_{0}$-holomorphic.

Acknowledgements. The author is very grateful to László Lempert for his encouragements and thanks Purdue University and The Banach Center, Warsaw for their support.

\section{REFERENCES}

[Ag] R. M. Aguilar, Dual canonical structures on tangent bundles of Riemannian manifolds, Preprint (1997).

[F-T] K. Furutani and R. Tanaka, A Kähler structure on the punctured cotangent bundle of complex and quaternion projective spaces and its application to geometric quantization I, J. Math. Kyoto Univ., 34-4 (1994), 719-737.

[F-Y] K. Furutani and S. Yoshizawa, A Kähler structure on the punctured cotangent bundle of complex and quaternion projective spaces and its application to geometric quantization II, Jap. J. Math., 21 (1995), 355-392.

[G-S] V. Guillemin and M. Stenzel, Grauert tubes and the homogeneous Monge-Ampère equation I, J. Diff. Geom., 34 (1991), 561-570.

[Ii] K. Ii, Kähler structures on tangent bundles of Riemannian manifolds of constant positive curvature, Preprint (1996).

[Ka] S. J. Kan, The asymptotic expansion of a CR invariant and Grauert tubes, Math. Ann., 304 (1996), 63-92.

[L-Sz] L. Lempert and R. Szőke, Global solutions of the homogeneous complex MongeAmpère equation and complex structures on the tangent bundle of Riemannian manifolds, Math. Ann., 290 (1991), 689-712.

[Ra1] J. H. Rawnsley, Coherent states and Kähler manifolds, Quart. J. Math. Oxford, 28 (1977), 403-415.

[Ra2] A nonunitary pairing of polarizations for the Kepler problem, Trans. Amer. Math. Soc., 250 (1979), 167-180.

[Sh] B. V. Shabat, An introduction to complex analysis, Nauka, Moscow, 1976, (Russian).

[So] J. M. Souriau, Sur la variété de Kepler, Symposia Math., 14, Academic Press, London (1974), pp. 343-360.

[Sz] R. Szőke, Complex structures on the tangent bundle of Riemannian manifolds, Math. Ann., 291 (1991), 409-428.

[Wo] N. M. J. Woodhouse, Geometric quantization, Oxford University Press, Oxford, 1992. 
1088 Budapest

Rákóczi u. 5

rszoke@cs.elte.hu 